ANUARIO DE Estudios MEdiEVAles

49/2, julio-diciembre de 2019, pp. 741-760

ISSN 0066-5061

https://doi.org/10.3989/aem.2019.49.2.13

\title{
EL USO DE HORAS DE RELOJ EN LAS CIUDADES DE CASTILLA. LOS EJEMPLOS DE PALENCIA Y VALLADOLID
}

\author{
THE USE OF CLOCK-HOURS IN THE CITIES OF CASTILE. \\ THE CASE STUDIES OF PALENCIA AND VALLADOLID
}

\author{
VÍCTOR PÉREZ ÁLVAREZ \\ Universidad de Valladolid \\ https://orcid.org/0000-0003-4230-3934
}

\begin{abstract}
Resumen: Las antiguas horas solares variaban de duración a lo largo del año y su recuento se iniciaba al amanecer y al anochecer. La llegada del reloj público a las ciudades y villas castellanas entre los siglos XIV y XV conlleva la introducción de las horas modernas o de reloj, que son de igual duración independientemente de la época del año y su recuento normalmente se comienza una sola vez al día. En este trabajo vamos a estudiar cómo se introducen estas nuevas horas de reloj en Castilla a través de los ejemplos de las ciudades de Palencia y Valladolid. Para ello explicaremos cómo conviven con las viejas horas solares y para qué actividades se emplean.
\end{abstract}

Palabras clave: reloj; hora; ciudad; campana; toque; mercado; concejo.

Abstract: Solar hour length changes with the season and its reckoning begins twice a day, from sunrise and from sunset. When public clocks appeared in Castilian cities and towns in the late $14^{\text {th }}$ and early $15^{\text {th }}$ centuries, modern clock hours were introduced. Their length is the same in all seasons and their reckoning begins only once a day. This paper studies how clock hours were introduced in Castile using Palencia and Valladolid as examples, and explains how modern clock hours coexisted with the old forms of time reckoning and what purposes they were used for.

Keywords: clock; hour; city; bell; ring; market; council.

\section{SUMARIO}

1. Introducción.-2. Palencia.-3. Valladolid.-4. Conclusiones.-5. Bibliografía citada.

\section{INTRODUCCIÓN ${ }^{1}$}

Antes de nada, hemos de aclarar qué entendemos por horas modernas o de reloj. Este último adjetivo implica la existencia previa de otra forma de

${ }^{1}$ Abreviaturas utilizadas: $\mathrm{ACP}=$ Archivo Catedralicio de Palencia; AGS $=$ Archivo General de Simancas; AHN = Archivo Histórico Nacional; AHPP = Archivo Histórico Provincial de Palencia; AHPV = Archivo Histórico Provincial de Valladolid; AMB = Archivo Municipal de Burgos; AMP = Archivo Municipal de Palencia; AMV = Archivo Municipal de Valladolid; ARCV = Archivo de la Real Chancillería de Valladolid; CSR = Casas y Sitios Reales; RGS = Registro General del Sello.

Citation / Cómo citar este artículo: Pérez Álvarez, Víctor (2019), El uso de horas de reloj en las ciudades de Castilla Los ejemplos de Palencia y Valladolid, "Anuario de Estudios Medievales" 49/2, pp. 741-760. https://doi.org/10.3989/ aem.2019.49.2.13

Copyright: (C) 2019 CSIC. Este es un artículo de acceso abierto distribuido bajo los términos de la licencia de uso y distribución Creative Commons Reconocimiento 4.0 Internacional (CC BY 4.0). 
dividir el día. Tal como el Prof. Dohrn-van Rossum señala, las divisiones antiguas del día presentan tres características fundamentales: en primer lugar, se divide el día y la noche de forma separada e independiente sin tener en cuenta la proporción entre uno y otro. En segundo lugar, cada uno de estos periodos se divide en doce partes llamadas horas, horas solares o desiguales, puesto que, como decimos, no se tiene en cuenta la estación del año. En tercer lugar, hay dos momentos de inicio del recuento que coinciden siempre con la salida y la puesta de $\mathrm{sol}^{2}$. Las "horas modernas" se pueden definir por contraposición a las "horas antiguas", ya que para obtenerlas se divide el conjunto día-noche en veinticuatro partes, es decir, en "horas iguales"3, cuya duración es siempre la misma independientemente de la estación del año. Además el recuento de horas modernas se inicia una sola vez al día, bien a la puesta de sol en el "reloj entero", o bien a medio día en el "medio reloj"s. Sólo en el llamado "gran reloj" o estilo de Núremberg se inicia el recuento dos veces al día, sin embargo es un sistema muy raro que se ha empleado en muy pocas ciudades, por lo se puede considerar la excepción que confirma la regla ${ }^{6}$.

El reloj mecánico aparece en Europa a finales del siglo XIII, probablemente en el Norte de Italia, aunque el uso de horas modernas está ligado a su difusión durante el siglo XIV, que, dependiendo de áreas, tiene lugar a partir de mediados o de finales del siglo. El profesor Dohrn-van Rossum ha establecido una cronología de difusión dividida en varios periodos. El primero de ellos abarca la primera mitad del siglo XIV, durante el cual se extiende por muchas de las grandes ciudades italianas. En la década de 1350 sale de Italia y los primeros relojes de edificio se construyen en grandes palacios y residencias principescas europeas. En el último cuarto del siglo las grandes ciudades europeas adquieren su primer reloj y aparecen los primeros relojeros locales, que aprenden el oficio de extranjeros, un hecho fundamental para la difusión de la nueva tecnología ${ }^{7}$. El caso de la Corona de Castilla, recientemente estudiado en una tesis doctoral defendida en la Universidad de Valladolid, encaja perfectamente en la cronología de difusión propuesta por el Prof. Dohrn, que también es aplicable a la Corona de Aragón. El primer reloj de edificio que conocemos

\footnotetext{
${ }^{2}$ Dohrn-van Rossum 2007, p. 152.

${ }^{3}$ También denominadas "equinocciales", puesto que en los días de los equinoccios tienen la misma duración que las horas solares.

${ }^{4}$ Forma de división del día en la que se cuentan las horas de una a veinticuatro desde después de puesto el sol.

${ }^{5}$ Estilo en el que se cuentan dos series de una a doce horas desde mediodía a media noche y desde media noche a mediodía, aunque la referencia para concertar el reloj es el mediodía.

${ }^{6}$ Sobre los diferentes sistemas de recuento horario moderno, véase Pérez Álvarez 2016, p. 15; 2015a, p. 46; Dohrn-van Rossum 2007, pp. 152-156; Bilfinger 1892, pp. 230-232.

${ }^{7}$ Dohrn-van Rossum 2007, p. 159.
} 
con seguridad en los reinos hispánicos fue el que ordenó construir Pedro IV de Aragón para su residencia del Castillo de Perpiñán en 1356. Los relojes públicos de las ciudades castellanas y aragonesas comenzaron a aparecer a finales de los años 1370 y en la década de 1380, como los de Salamanca, Valencia y Tortosa (1378), Sevilla, (1380-85)y Burgos (1384). Desde inicios del siglo XV ya los vemos en ciudades y villas de menor entidad, como por ejemplo Cuéllar (1403), Toro (antes de 1408) y Paredes de Nava (1422). A inicios del siglo XVI empezamos a documentar relojes públicos en villas y aldeas de pocos vecinos ${ }^{8}$.

Si bien los primeros relojes públicos castellanos datan del último cuarto del siglo XIV, no es hasta bien entrado el XV cuando comenzamos a documentar la utilización de horas modernas, sobre todo a partir de la segunda mitad, cuando las menciones dejan de ser aisladas y comienzan a ser más frecuentes.

Diversos historiadores a lo largo del siglo XX han debatido sobre su origen y difusión así como sus implicaciones socioculturales en la Europa de finales de la Edad Media9. Quienes consideran que la economía capitalista está ligada a un proceso de racionalización y organización del trabajo, incluyen en él la necesidad de un control del tiempo para la organización de la actividad diaria de las comunidades. Las comunidades monásticas serían las pioneras, puesto que están muy organizadas y sus miembros viven con una disciplina y una conciencia del valor del tiempo más acusada que el resto de la sociedad. Por este motivo, autores como Sombart o Mumford atribuyen a los monasterios la invención del reloj mecánico así como el germen de la llamada conciencia moderna del tiempo. Posteriormente, pasaría al entorno laico de las ciudades, que costearían la construcción de sus propios relojes públicos así como las torres cívicas para instalarlos. Los nuevos mercaderes a partir del siglo XIV, un grupo fundamentalmente urbano e imbuido de una mentalidad más lógica y racional, encontrarían atractivo el reloj y su nueva forma de indicar el tiempo con horas iguales. En esta línea se sitúa la clásica tesis de Jacques Le Goff, que contraponía el tiempo de los mercaderes, racional, laico, indicado y simbolizado por el reloj público, frente al tradicional tiempo laxo de las campanas y las horas canónicas, administrado por la iglesia. Para Le Goff, detrás de la tensión entre ambos había una lucha de poder entre la iglesia y las ciudades, además de un proceso de laicización de algunos aspectos de la vida Europea de finales de la Edad Media ${ }^{10}$.

\footnotetext{
${ }^{8}$ Para más detalles sobre la difusión del reloj público en Castilla con más ejemplos, véase Pérez Álvarez 2016, pp. 497-498.

${ }_{9}^{9}$ Véanse, por ejemplo, Le Goff 1983; Kula 1980; Gurievich 1990; Attali 1985; Whitrow 1990; Pomian 1994; Engammare 2004; Jaroszkiewicz 2016.

${ }^{10}$ Para una panorámica historiográfica, véase Dohrn-van Rossum 2007, pp. 10-15.
} 
Trabajos posteriores han demostrado que la supuesta necesidad de indicar el tiempo con más rigor en el ámbito urbano no ha sido el único motor para la difusión del reloj público, ya que es un accesorio de lujo y ostentación, que simboliza el poder y la autoridad del concejo. El reloj público en muchos casos no se difunde por iniciativa de las autoridades locales, sino por la de reyes o señores, que a veces incluso se encuentran con la oposición frontal de las autoridades locales, que se niegan a costearlos. También es habitual que se difunda por imitación, puesto que cuando el primer reloj llega a una localidad, las de alrededor desean tener el suyo propio ${ }^{11}$. Por tanto, para entender el fenómeno de la aparición y difusión del reloj en Europa en la Baja Edad Media, no sólo hay que tener en cuenta su papel como instrumento de indicación del tiempo, sino su valor simbólico. No obstante, puesto que esto último ya lo hemos estudiado en otras publicaciones ${ }^{12}$, en el presente trabajo vamos a centrarnos en lo primero.

En la Corona de Castilla son muy pocos los trabajos de investigación llevados a cabo sobre la introducción del reloj público, los cambios en la forma de indicar, administrar y concebir el tiempo asociados a él y su impacto sociocultural. En este artículo vamos a hacer un estudio aproximativo sobre el uso de las horas del reloj en el ámbito urbano de la Corona de Castilla a lo largo del siglo XV e inicios del XVI. En él tratamos de conocer para qué se utilizan las horas de reloj y cómo conviven con los sistemas antiguos de indicación del paso del tiempo. Hacer un trabajo extensivo superaría los propósitos de este artículo, por lo que hemos elegido los ejemplos de Palencia y Valladolid. La elección de estas dos ciudades se debe fundamentalmente a que para ellas disponemos de fuentes con datos útiles para realizar este estudio que no tenemos para otras ciudades. Palencia es particularmente interesante, puesto que conserva una serie de actas concejiles que comienza en 1421 y abarca casi todo el resto del siglo XV a penas sin lagunas, algo poco habitual en la Corona de Castilla. En Valladolid las actas no comienzan hasta $1497^{13}$, por lo que hemos tenido que recurrir a otro tipo de documentos, normalmente algo más tardíos que en el caso de Palencia. Uno de ellos es el llamado "Cronicón de Valladolid", que recoge acontecimientos acaecidos entre 1333 y 1539 y cuya particularidad es que utiliza con frecuencia horas de reloj para datarlos, sobre todo a partir de la segunda mitad del siglo $\mathrm{XV}^{14}$. De otras loca-

\footnotetext{
${ }^{11}$ Algo similar sucede en las villas de la Casa de Alba en el segundo cuarto del siglo XV; para más detalles sobre la difusión del reloj público en Castilla, Pérez Álvarez 2016.

${ }_{12}$ Pérez Álvarez 2016, pp. 497-498; véase además Pérez Álvarez 2013, 2015a, 2015b; Haber 1976; Maravall 1972; Mayr 1986.

${ }^{13}$ Arenzana 2016; agradecemos a su autor habernos facilitado su consulta.

${ }^{14}$ Sáinz de Baranda 1848.
} 
lidades castellanas tenemos muy pocos datos, normalmente sueltos, y rara vez disponemos de series documentales con información que nos den una visión de cómo se implantan las horas de reloj a lo largo del siglo XV. Burgos, por ejemplo, conserva actas concejiles y capitulares desde finales del siglo XIV, sin embargo no hemos podido incluirla ya que no hemos hallado referencias horarias en ellas.

Hay que tener en cuenta que la elección de sólo dos ejemplos puede distorsionar la visión del panorama general, por ello, las conclusiones de este estudio han de quedar sujetas a las de trabajos de investigación futuros, que, a pesar de que las fuentes son limitadas, podrán arrojar más luz sobre el tema.

\section{PALENCIA}

El concejo de la ciudad de Palencia mantiene un reloj público desde antes de 1421 en la Iglesia de San Miguel ${ }^{15}$. También hay reloj en la catedral de San Antolín desde antes de $1410^{16}$, probablemente desde el último cuarto del siglo XIV, aunque desconocemos si es un reloj público o si sólo está destinado al gobierno de la vida de los canónigos. El uso de horas de reloj se documenta casi tan temprano como la existencia del propio reloj. La mayor parte de las referencias las hemos encontrado en las actas concejiles y se emplean sobre todo para establecer las reuniones del concejo, para regular los horarios de mercados y para determinadas actividades industriales nocivas o molestas para los vecinos. También el cabildo empleaba las señales de su reloj como referencia para alguna de sus actividades, aunque menos que el concejo.

Las reuniones del concejo se pueden convocar de diferentes modos. En el acta más antigua conservada se especifica que se hizo a canpana rrepicada segund que es vso e costunbre ${ }^{17}$. En alguna otra ocasión posterior se hace siendo los oficiales llamados de antenoche ${ }^{18}$. Independientemente de esto, existían horarios previamente regulados para las reuniones, a veces en horas de reloj. La más antigua de estas normativas data de 4 de marzo de 1421 , cuando se establece que las reuniones de concejo se lleven a cabo lunes y viernes según uso y costumbre, entre las siete y las ocho y que los pleitos se

\footnotetext{
${ }^{15}$ Pérez Álvarez 2016, pp. 108-109.

${ }^{16}$ ACP, Armario III, Legajo IX $n^{\circ} 535, n^{\circ} 8$; citado en Reglero de la Fuente 2005-2006, p. 152.

${ }^{17}$ AHPP, R.02, Actas concejiles, f. 1r, Palencia, 1421, marzo, 2. Las actas concejiles de Palencia se custodian en su Archivo Municipal, sin embargo, consideramos que los absurdos trámites burocráticos que se nos exigía cumplimentar suponían una pérdida de tiempo, por lo que decidimos emplear las copias en microfilm del Archivo Histórico Provincial de Palencia.

${ }^{18}$ AHPP, R.02, Actas concejiles, f. 114r, Palencia, 1436, abril, 17.
} 
entendiesen entre las nueve y las diez ${ }^{19}$. Es, por tanto un testimonio bastante temprano del uso de horas de reloj. Un aspecto interesante de esta ordenanza es que sólo es válida para el periodo en que durase el entredicho que el obispo había impuesto a la ciudad con motivo de un conflicto por la corta de leña. El entredicho fue levantado el 2 de junio del mismo año ${ }^{20}$. Parece que esta situación obligaba o al menos motivaba a los regidores para utilizar el reloj para concertar las reuniones, aunque no es fácil precisar la razón concreta. Si el entredicho afecta a todos los habitantes de la ciudad, los oficios se hacen a puerta cerrada sólo para los religiosos, por lo que la ciudad puede quedar privada de ciertos toques de campanas. El acta en que consta la finalización del entredicho parece sugerir que se había suspendido el toque de las horas canónicas: este dia e ora, estando en capillas, se alço el enterdicho e tanieron las oras $^{21}$. Si precisamente algunos de los toques que desaparecen son los utilizados para convocar el cabildo, los oficiales del concejo estarían obligados a buscar otra señal pública para concertar la reunión. Sin embargo, durante este periodo encontramos algunas actas concejiles con referencias temporales a horas canónicas como prima, tercia o vísperas ${ }^{22} \cdot$ ¿Los toques de estas horas son los mismos que los que se tañeron al levantar el entredicho? Creemos que no, puesto que en el acta se asocia el toque al levantamiento del entredicho. Por otro lado el concejo se reúne habitualmente en el llamado palaçio de las capillas, en la iglesia de San Antolín ${ }^{23}$, lo cual pudo plantear un problema, ya que las personas afectadas por el entredicho no pueden asistir a los oficios divinos. Compartir el mismo espacio para dos actividades incompatibles quizá obligara a que el momento o el lugar de alguna de las dos tuvieran que ser modificados. No obstante, además de todas estas posibilidades, el uso del reloj en lugar de las campanas o las horas canónicas, puede tener la lectura simbólica de reafirmar la autoridad del propio concejo frente al cabildo en un momento de conflicto.

En cualquier caso en años posteriores existe una preocupación tanto por garantizar la asistencia de los regidores y alcaldes como para que lo hicieran a las horas establecidas y que acudiesen con puntualidad. Concretamente en 1436 se les recuerda su obligación de asistir y de hacerlo al tiempo lemita-

\footnotetext{
${ }^{19}$ AHPP, R.02, Actas concejiles, f. 3r, Palencia, 1421, marzo, 4; editado en: Fuente Pérez 1987, p. 193.

${ }^{20}$ AHPP, R.02, Actas concejiles, f. 13r, Palencia, 1421, junio, 2.

${ }^{21}$ Ibidem.

${ }_{22}$ AHPP, R.02, Actas concejiles, f. 7r, Palencia, 1421, abril, 11; ibidem, f. 10v, Palencia, 1421, mayo, 13; ibidem, f. 11r, Palencia, 1421, mayo, 14.

${ }^{23}$ Fuente Pérez 1987, p. 104.
} 
$d o^{24}$. En las reuniones de la semana siguiente constan las listas de los oficiales que no han asistido a ellas con puntualidad ${ }^{25}$. Tres décadas después, en 1469, fueron modificadas las horas de convocatoria mediante dos ordenanzas de ocho de marzo y de diez de octubre, que la establecen a las ocho y a las nueve, bajo pena de multas para quien llegase tarde o no se presentase ${ }^{26}$. Estos cambios de horarios son claramente estacionales, pues se realizan en primavera y otoño respectivamente. Es más, el primero de ellos coincide con el día exacto del equinoccio primaveral.

Las horas de reloj no sólo aparecen en ordenanzas, sino que se emplean en la vida real junto con otros tipos de referencias temporales como las horas canónicas ${ }^{27}$. Y no sólo se utilizan para establecer la convocatoria de las reuniones, sino también para datar otro tipo de actos. Así por ejemplo en 8 de febrero de 1422, a mitad de la reunión, después de tratar otros asuntos, consta como vna ora despues del medio dia, estando en el palaçio en el corredor el sol, se efectúa la entrega de los sellos de la ciudad al merino ${ }^{28}$. Se trata del palacio de las capillas donde se reúne el concejo habitualmente. En este caso, la referencia temporal es doble, ya que a la hora de reloj se añade la posición del sol, que debía de colarse en alguna dependencia orientada hacia el sur o suroeste y que quizá se utilizara como referencia para el paso del mediodía.

Fuera de las actividades de los oficiales del concejo, se emplean las horas del reloj para regular otras actividades de la ciudad, como la de los regatones de la fruta, que no podían comprarla antes de las doce de medio día ${ }^{29}$. También los del pescado, que si llegan el jueves a la ciudad no lo pueden comprar hasta el viernes a las nueve y si llegan el viernes u otro día de ayuno, hasta después medio día. Estos momentos se expresan en horas canónicas en 1455 y en horas de reloj dos años después ${ }^{30}$. Estas normas se imponían para evitar que unos pocos regatones acapararan mercancía con la consiguiente subida de precios y provocaran el desabastecimiento de la ciudad.

${ }^{24}$ AHPP, R.02, Actas concejiles, f. 113v, Palencia, 1436, abril, 11; citado en Fuente Pérez 1987, pp. 105-106.

${ }^{25}$ AHPP, R.02, Actas concejiles, f. 114r, Palencia, 1436, abril, 16 y 14.

${ }^{26}$ AHPP, R.03, Actas concejiles, Palencia, 1469, marzo, 6 y octubre, 17.

27 "Sabado seguiente a ora de prima" AHPP, R.02, Actas concejiles, f. 7r, Palencia, 1421, abril, 11; en "las capillas a ora de biesperas" AHPP, R.02, Actas concejiles, f. 22r, Palencia, 1421, agosto, 19; "En este dicho dia ante conçejo a ora de terçia" AHPP, R.02, Actas concejiles, f. 17r, Palencia, 1421 , julio, 4 .

${ }^{28}$ AHPP, R.02, Actas concejiles, f. 40v, Palencia, 1422, febrero, 18.

${ }^{29}$ AHPP, R.02, Actas concejiles, f. 121v, Palencia, 1436, julio, 11; citado en Esteban Recio 1989, p. 84, n. 205.

${ }^{30}$ AHPP, R.03, Actas concejiles, Palencia, 1455, marzo, 11; citado en Fuente Pérez 1987, pp. 73-74. 
En otra ocasión, en 1470, se emplea para limitar los horarios de trabajo de Juan de Fuentes, trapero y tintorero, cuya actividad es molesta y genera residuos nocivos para los vecinos de la ciudad. Se le autoriza a ejercer su oficio sólo después de las diez de la noche. Los días cercanos al solsticio de verano, los más largos del año, poco después de las nueve habría oscurecido por completo, por lo que quedaba garantizado que el trabajo se comenzaría todas las épocas del año bien avanzada la noche. Esta profesión solía ejercerse extramuros de la ciudad junto a algún curso de agua por la toxicidad de los productos químicos que se empleaban y los fuertes olores que desprendían. Sin embargo, Juan de Fuentes vivía en el barrio de Medina, en el centro de Palencia, lo que sin duda explica que sólo se le permita trabajar de noche, cuando todo el mundo estaría durmiendo en sus casas. Según se desprende del acta, todos los tintoreros de la ciudad tenían esta misma limitación ${ }^{31}$.

Como sucede en otras ciudades, en la noche es relativamente frecuente emplear horas de reloj, ya que se carece de la referencia del sol. En Palencia, además del trabajo de los tintoreros, hemos documentado su uso asociado al toque de queda al menos en dos casos. Uno de ellos, en 1455, para prohibir andar sin candela por la ciudad de noche después de las nueve o del toque de queda $^{32}$. La otra ocasión es más temprana, ya que data de finales de febrero de 1422, y es muy particular, puesto que se trata de una anotación del escribano del concejo en el libro de actas sobre la muerte de uno de sus hijos sólo cinco días después de nacer. Tanto el nacimiento como la muerte quedan registrados con la precisión del día y la hora. El nacimiento se produce el viernes veinte quando dio las nueue oras quando començaban a tanner terçia e la misa del dia en la iglesia de Sant Antolin ${ }^{33}$. El óbito es más impreciso, ya que se produce el miércoles 25 entre las siete y las ocho de la tarde, ya que se le entierra luego acabando de tañer la queda. En los libros de actas existen más anotaciones de defunciones ${ }^{34}$, aunque esta es la única en que se utilizan horas de reloj. También es la más particular de todas, puesto que es una anotación puramente personal, sólo importante para el escribano e intrascendente para el concejo de la ciudad. Sin duda el escribano aprovechó la oportunidad que le brindaba su cargo para dejar memoria de tan desgraciado hecho y de expresar el dolor de la pérdida de su hijo diciendo asy vaya la mia [anima] valdia commo la soya.

\footnotetext{
p. 60 .

${ }^{31}$ AMP, Actas concejiles, 1470, Palencia, 1470, enero, 12; citado en Fuente Pérez 1987,

${ }^{32}$ AMP, Actas concejiles, 1455, Palencia, 1455, abril, 22; citado en Esteban Recio 1989, p. 86.

${ }^{33}$ AHPP, R.02, Actas concejiles, f. 41r, Palencia, 1422, febrero, 20; editado en Fuente Pérez 1987, p. 28

${ }^{34}$ AHPP, R.02, Actas concejiles, f. 21r, Palencia, 1421, agosto, 12; f. 61v, Palencia, 1422, septiembre, 8 .
} 
Además del concejo, también el cabildo catedralicio utilizaba horas de reloj para regir sus propios asuntos, si bien hemos encontrado muchas menos referencias. La defunción del hijo del escribano es una posible referencia indirecta, puesto que, si coinciden las campanadas de las nueve de la mañana del reloj con la señal de tercia, con toda probabilidad es porque el toque manual de tercia se efectúa con la referencia del reloj. No hemos hallado en las actas capitulares ninguna noticia para esta época que así lo establezca, pero el testimonio indirecto del escribano del concejo lo pone de manifiesto. En julio de 1459 sí que documentamos el uso del reloj como referencia para los toques canónicos, ya que entonces se ordenó que se tañese a maitines una hora después de media noche, a nona a las dos y a prima a la hora que el cabildo mandase ${ }^{35}$.

\section{VALLADOLID}

Al igual que en Palencia, desconocemos la fecha de instalación del primer reloj público de Valladolid, que debió de ser anterior a 1437, año de su primera mención ${ }^{36}$, aunque posiblemente date de inicios del siglo XV o de finales del XIV. En aquel momento estaría instalado en la torre de la colegiata de Santa María la Mayor. Para 1525, y quizá desde mediados del siglo XV, había reloj en San Benito El Real, si bien desconocemos si era público o sólo para uso del monasterio ${ }^{37}$. En 1497 el Regimiento ordenó construir otro reloj público en el monasterio de San Francisco, en la plaza y mercado mayor de la villa ${ }^{38}$.

Del siglo XV tenemos muy pocas referencias sobre el uso de horas modernas en Valladolid, que hasta la segunda mitad de este siglo son aisladas. Algunas de ellas son además las primeras pruebas de la existencia de reloj mecánico en Valladolid. En 1425 nacía el que sería Enrique IV y el Cronicón registra el hecho en horas de reloj. Para esta fecha es más que probable que se empleasen relojes mecánicos en el ámbito de la $\operatorname{corte}^{39}$, por lo que esta hora, en caso de ser real, no tendría por qué haberse determinado necesariamente con un reloj público. Es más, cabe la posibilidad de que fuera calculada por un astrólogo mediante un astrolabio u otro instrumento para poder levantar el horóscopo del recién nacido. En cualquier caso, independientemente del

\footnotetext{
${ }^{35}$ ACP, Actas capitulares, Palencia, 1459, julio, 17; citado en Polanco Pérez 2008, p. 284.

${ }^{36}$ Rucquoi 1997, p. 371.

${ }^{37}$ Pérez Álvarez 2016, pp. 161-163.

${ }^{38}$ Ibidem, p. 156.

${ }^{39}$ Pérez Álvarez 2013, pp. 494-495.
} 
instrumento utilizado, insistimos que de ser la noticia cierta, quedaría atestiguado por primera vez el uso de horas de reloj en Valladolid, aunque no su indicación pública.

Después del nacimiento de Enrique IV recogido por el Cronicón, la siguiente referencia temporal moderna data de 1437, cuando la audiencia convoca a un testigo para mañana a las nueve horas del relox antes de medio $d i a^{40}$. Este documento ya lo hemos citado anteriormente y su valor es mayor, puesto que es contemporáneo del hecho que describe, al contrario que la noticia del nacimiento de Enrique IV del Cronicón. En este caso sí que podemos estar ante una indicación pública del tiempo, puesto que la audiencia es una institución pública y la señal de la hora tiene validez en tanto en cuanto es percibida por la colectividad. Esta breve expresión, decíamos, muestra la muy probable existencia de reloj público en Valladolid. Lo que demuestra con total certeza es que se conocían las horas modernas y que se utilizaban, al menos en aquella fecha. Sin embargo su uso seguramente no sería del todo habitual, puesto que se especifica que son horas del relox. Las nueve horas antes de medio día son equivalentes a la hora de tercia, hora canónica a la que la audiencia de Valladolid se reúne normalmente. Esta noticia es aislada y tenemos que esperar hasta 1456 para hallar otro testimonio de uso de horas modernas o de reloj, cuando los monjes de San Benito el Real salen de viaje de Valladolid a las dos e media despues de media noche $e^{41}$. Este documento guarda similitudes con el Cronicón ya que está conformado por una sucesión de apuntes de hechos o anécdotas ordenados cronológicamente. También en varios de ellos se especifica el momento del día en que tienen lugar, de los cuales unos están expresados en horas canónicas y otros en horas modernas. Además de la posible presencia de relojes mecánicos en otros lugares, este documento muestra cómo los benedictinos de Valladolid ya estaban familiarizados con las horas modernas.

Después de este relato del viaje tenemos que volver al Cronicón ${ }^{42}$. Además del nacimiento de Enrique IV en 1425, lo que más nos interesan son las noticias de hechos acaecidos en Valladolid de los años sesenta en adelante, es decir, los acaecidos en vida de su autor, el Dr. Toledo. La más antigua que con más probabilidad vivió es el nacimiento de su hijo, el bachiller Diego de Alcaraz, en trece de junio de 1461 nueve horas y terçia despues de medio

\footnotetext{
${ }^{40}$ Rucquoi 1997, p. 371.

${ }^{41}$ AHN, Clero secular-regular, leg. 7731, n ${ }^{\circ}$ 58,"rrelaçion de las cosas que fezimos en el camino", 1456; citado en Pérez Álvarez 2007, n XXXI, p. 142.

${ }^{42} \mathrm{Su}$ autor, el Doctor Toledo, residía en Valladolid, donde murió en 1497; Sáinz de Baranda 1848, p. 9.
} 
$d i a^{43}$. Es precisamente el periodo que coincide con su vida en el que más referencias temporales en horas de reloj aparecen, lo que indica que su autor estaba muy familiarizado con ellas.

De esta época, además del testimonio del Dr. Toledo, tenemos algún documento suelto más, aunque no aporta demasiado conocimiento adicional sobre la implantación de las horas de reloj en Valladolid. Sin duda la mejor fuente para conocer esto son las actas concejiles, ya que las ordenanzas con regulaciones horarias para actividades del común, obligan a la población a prestar atención a las señales horarias, lo que sin duda contribuye a la implantación del uso del reloj mecánico en sectores amplios de la población. Un ejemplo lo encontramos en la regulación de la renta de la sal, cuyo remate es establecido por el concejo el jueves a las 12 del mediodía, a la última campanada del reloj de Santa María ${ }^{44}$. Sin embargo, una vez más hay que lamentar que las actas concejiles no empiecen hasta 1497, ya que es a partir de este momento cuando, gracias a ellas, se multiplican las referencias, en este caso sobre su uso por parte del regimiento.

Lo habitual es que se utilicen para la convocatoria de las reuniones de órganos públicos. Aunque esto no implica que dejara de usarse la antigua campana de San Miguel, probablemente el primer instrumento del concejo para emitir señales. Así, en julio de 1499 quedan en reunirse entre las syete y las ocho en el monesterio de Sant Francisco para inspeccionar las casas de los boticarios, ya que algunos de ellos estaban expendiendo medicinas fraudulentas ${ }^{45}$. Unos días después se ordena que esta tarde para las tres oras de despues de medio dia se junten en Sanct Miguel los que tienen las llaves del archivo para que saquen determinado documento ${ }^{46}$. En otra ocasión, en 1506, quedan en reunirse en la posada del dicho señor corregidor a las quatro oras despues de medio dia para organizar una muestra de apoyo de la villa en favor de la reina Juana $^{47}$. La asistencia a las reuniones del regimiento, tal como recoge Pino Rebolledo, no siempre era la deseada, problema que el corregidor trata de solucionar en más de una ocasión imponiendo un horario para las reuniones y multas a quienes se ausenten de ellas sin justificación. En 1499 tenemos constancia por primera vez de este asunto ${ }^{48}$. En 1503 el corregidor establece que los regidores viniesen a rregimiento andando las nueve ${ }^{49}$. En 1519 se establece que desde

\footnotetext{
${ }^{43}$ Ibidem, p. 49.

${ }^{44}$ AMV, Actas concejiles, $n^{\circ}$ 2, ff. 554r-555r, Valladolid, 1511, julio, 7.

${ }^{45}$ AMV, Actas concejiles no 1, f. 237r, Valladolid, 1499, julio, 3.

${ }^{46}$ AMV, Actas concejiles no 1, ff. 237-240r, Valladolid, 1499, julio, 7

${ }^{47}$ AMV, Actas concejiles, $n^{\circ}$ 2, f. 263r, Valladolid, 1506, octubre, 30.

${ }^{48}$ AMV, Actas concejiles $n^{\circ}$ 1, f. 240r, Valladolid, 1499, julio, 10.

${ }^{49} \mathrm{AMV}$, Actas concejiles $\mathrm{n}^{\mathrm{o}}$ 2, f. 89v, Valladolid, 1503, noviembre, 15.
} 
octubre hasta Pascua Florida acudan a las nueve de la mañana y desde entonces hasta septiembre a las ocho; en 1520 el horario de invierno queda igual pero el de verano se adelanta una hora ${ }^{50}$. En algunas ocasiones de nada debían de servir estas ordenanzas, como en la reunión de tres de octubre de 1519, en la que, a pesar de que habían dado las diez en el reloj de San Francisco, estaban solos el corregidor y el regidor Verdesoto ${ }^{51}$. No se podía hacer regimiento cuando había menos de tres regidores, por lo que el elevado absentismo obligaba a suspender algunas reuniones. El problema se documenta de nuevo décadas más adelante, como en 1542 o en 1552. En esta última ocasión el corregidor actuó con severidad y convocó a los regidores para esa misma tarde a las dos so pena de mil ducados a quien no apareciese $e^{52}$, una multa de cuantía muy elevada. A medida que avanzaban los años la puntualidad de los regidores se iba haciendo más importante, por ello, para controlar con fiabilidad las horas a las que entraban y salían del concejo, en 1551 se ordena la compra de un reloj pequeño para la sala del regimiento, ya que con la mucha gente que concurre en la plaça no se oye en la sala el rrelox de San Francisco ${ }^{53}$.

Además de para las reuniones del concejo, veíamos como en 1437 se empleaban horas de reloj en la audiencia y a finales del siglo XV, en las ordenanzas de los Reyes Católicos, se establecen horarios de reloj en las audiencias de la Chancillería. Independientemente de que esto último se llevara a término o no, queda claro que cada vez es más habitual usar el reloj para reunir a los miembros de órganos colegiados como los que hemos utilizado en los ejemplos.

Además de para convocar reuniones, las horas de reloj se utilizan para regular el tiempo de ciertas actividades comerciales. Así por ejemplo, se limita el horario de compra al por mayor de determinados productos con el fin de que nadie los acapare y suban los precios. Las personas dedicadas a esta actividad son los regatones y en 1497 se prohíbe a los de la fruta que la compren a personas de fuera de la villa hasta el día siguiente de que llegaren, a las nueve de la mañana en verano o hasta las once en invierno ${ }^{54}$. En $1551 \mathrm{el}$ horario era algo diferente, puesto que la hora límite en verano eran las once de la mañana, pero como medida excepcional hubo de adelantarse a las nueve durante los meses de julio y agosto, puesto que el calor estropeaba la fruta en las horas centrales del día ${ }^{55}$.

\footnotetext{
${ }^{50}$ Pino Rebolledo 1990, p. 8.

${ }^{51}$ AMV, Actas concejiles no 4, f. 577v, Valladolid, 1519, octubre, 3.

52 Pino Rebolledo 1990, p. 11.

${ }^{53}$ AMV, Actas concejiles n ${ }^{\circ}$ 7, f. 26r, Valladolid, 1551, abril, 22.

${ }^{54}$ AMV, Actas concejiles n ${ }^{\circ}$ 1, f. 421, Valladolid, 1497, noviembre, 21.

${ }^{55}$ AMV, Actas concejiles $n^{\circ}$ 7, f. 42v, Valladolid, 1551, julio, 8.
} 
Algo similar se estableció para los moros obligados al fuego en 1497, a los que se les permitió comprar madera para sus casas los martes después de las diez de la mañana entre los meses de abril a septiembre y a partir de las once durante la otra mitad del año so pena de seis mil maravedíes y perder la madera, la cual se la repartirían entre los fieles de la villa y el acusador ${ }^{56}$. Los moros debieron de sentirse perjudicados por esta medida, así que en 1501 fueron ante la Cámara para protestar y exigir que esta medida se aplicase sólo a quienes especulaban con la madera comprando grandes cantidades para luego revenderla a precios más elevados. La Cámara tuvo en cuenta la petición de la aljama de Valladolid y estableció que la ordenanza se aplicara sólo a los regatones de la madera ${ }^{57}$. Para entonces la ordenanza concejil debía de haberse modificado, puesto que la restricción tenía lugar desde el lunes a medio día hasta el miércoles a la hora de tercia.

Otro aspecto de la vida vallisoletana que el concejo regula mediante el reloj es la apertura y cierre de las puertas de la muralla. En este caso el sistema horario empleado es mixto, puesto que la apertura se efectúa al toque del esquilón de la misa del alba de San Francisco y el cierre a las nueve de la noche entre septiembre y marzo y a las diez el otro medio año, que coincide con el toque de queda en la campana de San Miguel ${ }^{58}$. El cierre nocturno de las puertas no sólo es necesario para la seguridad de la villa, sino también para garantizar el cobro de impuestos a las mercadurías que se vendían en ella y que procedían del exterior ${ }^{59}$.

Las horas del reloj también se emplean en algunas ocasiones para determinar el inicio y el fin de la jornada laboral de algunos trabajadores, si bien de forma muy puntual. Lo habitual es trabajar desde el amanecer hasta el anochecer haciendo tan sólo un descanso a medio día para comer, o trabajar media jornada, bien de mañana o bien de tarde. Un ejemplo es el de Juan Lazo, que al menos entre 1497 y 1498 limpia la villa con dos carros cherriones y cuatro mozos, cuya jornada según el contrato que había hecho con el regimiento, empieza a las siete de la mañana entre abril y septiembre y a las ocho el otro medio año, para acabar después de puesto el sol, pudiéndose tomar una hora de descanso para comer. Si algún día no realizara su trabajo o marchare antes de puesto el sol perdería el jornal completo de ese día ${ }^{60}$. En otro caso en 1501, se paga a varios obreros por su trabajo hasta las

\footnotetext{
${ }^{56}$ AMV, Actas concejiles no 1, f. 301, Valladolid, 1497, julio, 19.

${ }^{57}$ AGS, RGS, leg. 150105, $\mathrm{n}^{\circ} 33$, Valladolid, 1501, mayo, 8.

${ }^{58}$ AMV, Actas concejiles $n^{\circ}$ 2, f. 90r, Valladolid, 1503, marzo, 27; citado en Domínguez Rodríguez 1976, p. 7.

${ }^{59}$ AMV, Actas concejiles, $\mathrm{n}^{\mathrm{o}}$ 2, f. 515v, Valladolid, 1511, febrero, 7.

${ }^{60}$ AMV, Actas concejiles n ${ }^{\circ}$ 1, f. 210r, Valladolid, 1499, abril, 2.
} 
diez del día en la puente de la Solanilla y reciben cada uno siete maravedíes. En ninguno de estos dos casos los obreros cobran el salario por horas, sino por días o medios días.

Decíamos al principio que las señales temporales previas a la existencia de reloj público en Valladolid y al recuento de horas moderno no desaparecen, sino que conviven con el reloj mecánico durante todo el Antiguo Régimen y que además algunas de ellas hasta se someten a él. Es el caso del toque de queda a principios del siglo XVI, que ya no se hace cuando el campanero considera que ha oscurecido el día, sino desde las nueve y media fasta que sean dadas las diez ${ }^{61}$. Así, no sólo se regula el momento exacto del toque, sino también su duración. Aunque el toque de queda perviva hasta el siglo XIX, esto no hace más que confirmar la primacía del reloj sobre la vieja señal.

Además de para cuestiones de buen gobierno de la villa, el reloj y sus referencias temporales también se utilizan a veces en la data de documentos, en crónicas y anales o en general en el registro de hechos considerados relevantes. Antes de la aparición e implantación del reloj mecánico en tierras castellanas esta práctica existía empleando otras referencias, frecuentemente horas canónicas, pero lo que aquí nos interesan son los documentos en los que se emplean horas de reloj, puesto que, si bien no es una práctica totalmente nueva, sí que representa un cambio y nos muestra el grado de implantación de las horas modernas de reloj. La primera vez que vemos esto en Valladolid es en el ya citado documento de los frailes de San Benito de 1456 en el que se registran algunos hitos de su viaje en horas de reloj, como la salida del monasterio a las dos y media, justo antes de amanecer. También tenemos el caso de Catalina López, la qual fino domingo xvi dias de febrero de lxxvii antes que amanesçiese dos horas ${ }^{62}$. Lo más notable aquí es sin duda el Cronicón de Valladolid, en el que el Doctor Toledo registra en horas de reloj diversos hechos acaecidos en Valladolid en la segunda mitad del siglo XV y vividos por él. Este tipo de noticias y de empleo de las horas de reloj es típico pero no exclusivo de crónicas o de anales. A veces en las actas concejiles vemos cómo se registran hechos en horas de reloj para fines similares que poco o nada tienen que ver con el buen gobierno o la coordinación de diferentes personas o colectivos. Así, en las actas concejiles de Valladolid queda registrado el fallecimiento de la reina Isabel La Católica en Medina del Campo a las once antes de mediodía ${ }^{63}$. Unos días después del acceso al trono de la reina Juana I

\footnotetext{
${ }^{61} \mathrm{AMV}$, Actas concejiles $\mathrm{n}^{\circ}$ 2, f. 9v, Valladolid, 1502, febrero; AMV, Actas concejiles $\mathrm{n}^{\circ} 2$, f. 43v, Valladolid, 1502, agosto, 3.

${ }^{62}$ AHN, Clero secular-regular, leg. 7716, Las mandas de Catalina Lopez de Leon... 1477.

${ }^{63}$ AMV, Actas concejiles, ${ }^{\circ}$ 2, f. 187v, Valladolid, 1504, noviembre, 27.
} 
de Castilla, queda registrado cómo en la villa, por orden de Fernando el Católico, se alzaron pendones por la reina a las tres de la tarde ${ }^{64}$. Algo similar encontramos en otro tipo de documentos, entre ellos en una relación de las obras efectuadas en el claustro de San Benito el Real en $1545^{65}$.

Gracias a las actas concejiles, las señales públicas, así como su uso para el gobierno público, es el que mejor conocemos. Escasas sin embargo son las noticias que podemos obtener sobre el conocimiento del tiempo por parte de personas particulares, sea con estas señales públicas o con instrumentos propios. La posesión de relojes u otros instrumentos de indicación del tiempo por parte de particulares puede estar en relación con una necesidad de conocer el paso de las horas. A mediados del siglo XV existían relojes portátiles pequeños en la corte, no siempre mecánicos, como el que Enrique IV regaló a su esposa en 1462 y fue adquirido de un mercader de Valladolid llamado Ubert por sesenta maravedíes ${ }^{66}$. Si el rey había comprado este objeto, otros miembros de la corte también podrían haber adquirido otros similares, más aun teniendo en cuenta que su precio no era demasiado elevado. No hemos podido constatar esto último, y menos aún si este tipo de instrumentos era o no habitual entre los particulares en esta época. Además de esta cuestión no resuelta, aún habría que plantearse si, en caso de haber estado extendidos, realmente eran utilizados para conocer la hora o como complementos del vestido, del ajuar doméstico o como elementos de ostentación. Por tanto no sabemos si en Valladolid en el siglo XV había personas con la necesidad o el deseo de disponer de sus propios instrumentos para conocer la hora.

En cualquier caso, independientemente de que poseyera reloj particular o no, conocemos el caso de una persona que estaba muy familiarizada con el recuento horario moderno. Nos referimos al Dr. Toledo, autor principal del Cronicón, que, como hemos avanzado, debió de estar con seguridad en Valladolid entre 1461, cuando nació su hijo, quizá desde unos años antes, hasta su fallecimiento en 1497. La mayor parte de los hechos que recoge en su relación están registrados con hora de reloj, lo que demuestra que tenía un concepto moderno del tiempo y que utilizaba las horas de reloj para un fin particular, la redacción de su crónica.

Otro caso de gran interés es el de Maestre Alonso, un relojero que murió en Valladolid en $1511^{67}$, en cuyo inventario de bienes post mortem

\footnotetext{
${ }^{64} \mathrm{AMV}$, Actas concejiles, $\mathrm{n}^{\mathrm{O}}$ 2, f. 187vValladolid, 1504, diciembre, 6.

${ }^{65}$ AHN, Clero secular-regular, leg. 7709, s/n, 1545.

${ }^{66}$ AGS, CSR, leg. 97, f. 236r.

${ }^{67}$ Para conocer la vida y la trayectoria profesional de este relojero, véase Pérez Álvarez 2016, pp. 350-356.
} 
aparece un despertador en la camara donde dormian ellos ${ }^{68}$, es decir, el relojero y su esposa, Mari Rodríguez de Oviedo. Esta noticia es de gran interés, ya que el objeto aparece contextualizado, lo cual nos ayuda a identificar su posible función, que en este caso sería la de despertar por la mañana al matrimonio. A priori podemos pensar que si fuera un elemento de ostentación no estaría en el dormitorio, sino en otro lugar de la casa más visible a la gente de fuera. Estamos, por tanto, ante un instrumento particular de indicación del tiempo. Sin embargo, el uso de un despertador por la mañana no tiene porqué implicar una conciencia muy desarrollada del tiempo, ya que su función es la de emitir una señal en un momento determinado, no la de especificar la hora concreta, ni mucho menos la de tañer todas las horas creando una secuencia temporal a lo largo del día. No obstante, es un elemento de gran interés, ya que sólo lo hemos hallado en este inventario de principios del siglo XVI. Por otro lado, un despertador simple es relativamente sencillo de construir y requiere muy poca cantidad de hierro, si a ello añadimos que su posesor era maestro relojero, comprenderemos que el esfuerzo económico que realizaría para tenerlo no sería demasiado importante.

Si avanzamos en el siglo XVI sí que encontramos más relojes pequeños, tanto mecánicos como de sol. El mercader Sansín de Villanueva, que falleció en 1554, tenía entre sus mercadurías, los siguientes relojes ${ }^{69}$ :

- quatro dozenas y diez espejos dazero con rrelox

- tres gruesas de rrelox quadrados de madera

- una gruesa y media de rrelox redondos de madera

- tres dozenas de relox de marfil pequeños

- vn rrelox de marfil dAlemania

- dos rrelox de marfil echura de Ynglaterra

- vna dozena y media de rrelox de marfil quadrados

Como el propio Bennassar señala, no es fácil saber exactamente qué son algunos de los objetos contenidos en el inventario. De los relojes, que en total sumarían la desorbitada cifra de ochocientos trece, lo único que parece seguro es que ninguno de ellos era mecánico. Los de marfil, especialmente el de Alemania, debieron ser de sol, como los que en el siglo XVI se construían en Núremberg o Augsburgo. Los de madera cuadrados y redondos que hacen unos setecientos cincuenta en total, pueden ser también de sol, más económicos que los de marfil. Los que desconocemos por completo que eran son los espejos de acero con reloj. Si damos por hecho que, salvo estos últimos, eran

\footnotetext{
${ }^{68}$ ARCV, Pleitos civiles, Varela (F), Caja 2061-1, f. 63v, Valladolid, 1511, mayo, 15.

${ }^{69}$ AHPV, leg. 51, ff. 572r-578v, Valladolid, 1554, marzo, 31; citado en Bennassar 1989, p. 38 .
} 
todos de sol, el número de ellos era altísimo, por lo que también su consumo debía de ser importante. Por su abundancia es posible que la mayoría de ellos fueran muy económicos y por tanto menos susceptibles de ser utilizados como elementos de ostentación. Es posible que los de marfil, más escasos en número, fueran más valiosos, ya que tan sólo hay veintiuno, de ellos especialmente los dos de echura de Ynglaterra y el único dAlemania. Sea como sea, este inventario de bienes de Sansín de Villanueva nos puede dar pistas sobre los objetos de consumo en el Valladolid de mediados del siglo XVI, pues los relojes que vendía estarían seguramente al alcance de un público amplio, ya que por su elevadísimo número, especialmente los de madera, no debían de ser muy caros. Sin embargo, esto tampoco es prueba determinante de que existiera una necesidad generalizada entre los vallisoletanos de un amplio rango socioeconómico de conocer las horas a nivel particular, aunque sin duda la disponibilidad de relojes a bajo precio facilitaría de alguna forma una mayor familiarización con el recuento de horas moderno y por tanto impulsaría una mayor conciencia del tiempo.

\section{CONCLUSIONES}

En los ejemplos de Valladolid y de Palencia que hemos estudiado, vemos que al menos desde la primera mitad del siglo XV se emplean horas de reloj para diversos fines. Las referencias van aumentando conforme avanza el siglo, especialmente en su segunda mitad, sin embargo no sólo puede que se deban a un mayor grado de implantación de las horas modernas, sino a la mayor disponibilidad de fuentes. Esto se hace más patente en el caso de Valladolid, ciudad que no conserva actas concejiles anteriores a 1497. Si tenemos en cuenta que en este año las referencias son numerosas y la cronología de las referencias extraídas de las actas palentinas, podemos considerar muy probable que, de haberse conservado actas en Valladolid para todo el siglo XV, hubiéramos hallado muchas más referencias horarias en años anteriores.

Es evidente que si se citan horas de reloj es porque se conocen y se emplean con mayor o menor difusión, sin embargo, la ausencia de citas en la documentación no necesariamente implica que no se conozcan o no se empleen. Sirva como ejemplo la ciudad de Burgos, que conserva excelentes archivos con series de actas capitulares en la catedral y de actas concejiles en el Archivo Municipal que comienzan a finales del siglo XIV y apenas tienen lagunas en los siglos siguientes. Por otro lado, sabemos que el arzobispo promovió la construcción de un reloj público en 1384 con una gran campana, a cuya financiación el concejo contribuyó en parte. Éste reloj estuvo en 
funcionamiento a lo largo de todo el siglo XV, puesto que en el Archivo Catedralicio están registrados los pagos de los salarios de sus cuidadores ${ }^{70}$. Sin embargo la primera mención sobre su uso por parte del concejo data de $1426^{71}$ y después de ella tenemos que esperar varias décadas hasta documentar la próxima. Resulta raro pensar que en una ciudad como Burgos, con una importante actividad comercial y artesanal, que desde 1384 dispone de reloj público, no conociera y empleara horas modernas en su día a día desde época temprana. La incógnita queda abierta, a menos que aparezcan fuentes que lo aclaren. Paul Glennie y Nigel Thrift, en su estudio sobre el uso de horas de reloj en Inglaterra, señalan que antes de la Revolución Industrial, el uso de horas modernas estaba más extendido de lo que a priori podemos pensar ${ }^{72}$. Estos autores llaman la atención sobre el silencio de las fuentes al respecto en épocas tempranas, lo que ha llevado, según ellos, a tener una visión distorsionada sobre la cronología de la implantación de las horas de reloj, que debió de ser mucho mayor de lo que a priori las fuentes indican.

\section{BIBLIOGRAFÍA CITADA}

Arenzana Antoñanzas, Víctor (2016), Libros de actas del concejo de Valladolid (1497-1520), Valladolid, Universidad de Valladolid (tesis doctoral).

Attali, Jacques (1985), Historias del tiempo, México, Fondo de Cultura Económica.

Bennassar, Bartolomé (1989), Valladolid en el Siglo de Oro: una ciudad de Castilla y su entorno agrario en el Siglo XVI, Valladolid, Ámbito.

Bilfinger, Gustav (1892), Die Mittelalterlichen Horen und die modernen Stunden, Stuttgart, Verlag von W. Kohlhammer.

Dohrn-van Rossum, Gerhard (2007), Geschichte der Stunde. Uhren und moderne Zeitordnungen, Colonia, Anaconda Verlag.

Domínguez Rodríguez, Ana (1976), Aspectos del urbanismo vallisoletano en torno al año 1500: puertas, arrabales y puentes, Madrid, Instituto de Geografía Aplicada del Patronato "Alonso de Herrera".

Engammare, Max (2004), L'ordre de temps. L'invention de la ponctualité au $X V I^{e}$ siècle, Ginebra, Droz.

Esteban Recio, Asunción (1989), Palencia a fines de la Edad Media. Una ciudad de señorío episcopal, Valladolid, Universidad de Valladolid.

${ }^{70}$ Pérez Álvarez 2016, pp. 128-133.

${ }^{71}$ AMB, Actas concejiles, $n^{\circ}$ 5, f. 41v, Burgos, 1426, octubre, 29.

${ }^{72}$ Glennie, Thrift 2009, p. 17. 
Fuente Pérez, María Jesús (1987), Palencia. Cien años de vida y gobierno de la ciudad a través de las actas municipales (1421-1521), Palencia, Diputación Provincial.

Glennie, Paul; Thrift, Nigel (2009), Shaping the day. A History of Timekeeping in England and Wales 1300-1800, Oxford, Oxford University Press.

Gurievich, Arón (1990), Las categorías de la cultura medieval, Madrid, Taurus.

Haber, Francis C. (1976), The Cathedral Clock and the Cosmological Clock Metaphor, en Fraser, Julius Thomas; Lawrence, Nathaniel (eds.), The Study of Time II. Proceedings of the Second Conference of the International Society for the Study of Time, Nueva York, Springer-Verlag.

Jaroszkiewicz, George (2016), Images of Time: Mind, Science, Reality, Oxford, Oxford University Press.

Kula, Witold (1980), Las medidas y los hombres, Madrid, Siglo XXI.

Le Goff, Jacques (1983), Tiempo, trabajo y cultura en el occidente medieval, Madrid, Taurus.

Maravall, José Antonio (1972), Estado moderno y mentalidad social. Siglos XV al XVII, Madrid, Revista de Occidente.

Mayr, Otto (1986), Authority, Liberty \& Automatic Machinery in Early Modern Europe, Baltimore, John Hopkins University Press

Pérez Álvarez, Víctor (2007), Concepción moderna del tiempo en el viaje de unos frailes de San Benito el Real de Valladolid a mediados del siglo XV, "Miscelánea Medieval Murciana" 31, pp. 139-148.

Pérez Álvarez, Víctor (2013), Mechanical Clocks in the Medieval Castilian Royal Court, “Antiquarian Horology" 34/4, pp. 489-502.

Pérez Álvarez, Víctor (2015a), El descubrimiento y la difusión del reloj mecánico en la Europa bajomedieval. Una perspectiva general, en Criado Mainar, Jesús; Borque Ramón, Juan José (coords.), El Relox viejo de Veruela. Un testimonio de la relojería mecánica bajomedieval, Zaragoza, Diputación de Zaragoza - Institución Fernando El Católico.

Pérez Álvarez, Víctor (2015b), The Role of the Mechanical Clock in Medieval Science, "Endeavour" 39/1, pp. 63-68.

Pérez Álvarez, Víctor (2016), Técnica, tiempo y ornato: el reloj público en Castilla entre los siglos XIV-XVI, Valladolid, Universidad de Valladolid (tesis doctoral).

Pino Rebolledo, Fernando (1990), El primer libro de actas del ayuntamiento de Valladolid año 1497, Valladolid, Ayuntamiento de Valladolid.

Polanco Pérez, Arturo (2008), La catedral de Palencia en el siglo XV (1402-1470). Poder y comportamientos sociales a finales de la Edad Media, Palencia, Diputación de Palencia - Institución Tello Téllez de Meneses. 
Pomian, Krzysztof (1994), El orden del tiempo, Gijón, Júcar.

Reglero de la Fuente, Carlos (2005-2006), La iglesia catedral de Palencia en el siglo XIV (1313-1379): crisis y reformas, "Edad Media. Revista de historia" 7 , pp. 121-160.

Rucquoi, Adeline (1997), Valladolid en la Edad media. 2. El mundo abreviado, Valladolid, Junta de Castilla y León, Consejería de Educación y Cultura,

Sáinz de Baranda, Pedro (1848), Cronicón de Valladolid, Madrid, Imprenta de la viuda de Calero.

Whitrow, Gerald James (1990), El tiempo en la historia: la evolución de nuestro sentido del tiempo y de la perspectiva temporal, Barcelona, Crítica.

Fecha de recepción del artículo: junio 2017

Fecha de aceptación y versión final: febrero 2018 